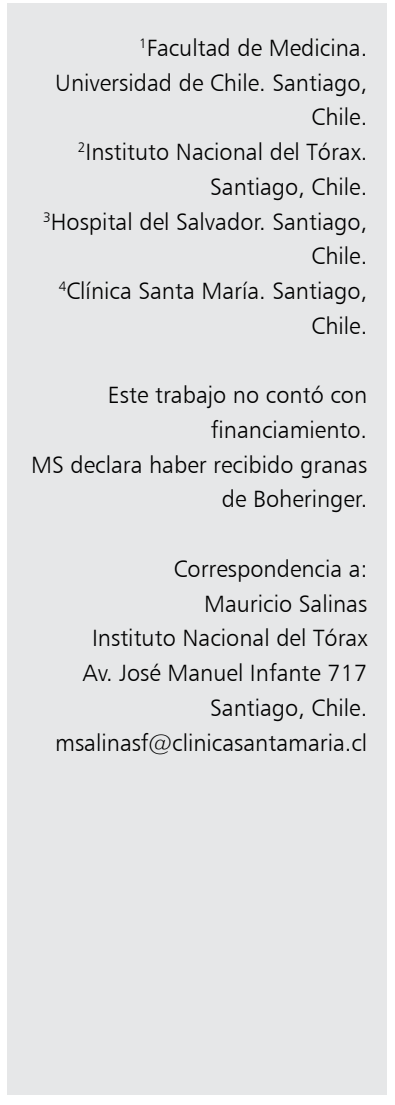

\section{Enfermedades pulmonares intersticiales. Una perspectiva actual}

\author{
MAURICIO SALINAS ${ }^{1,4}$, MATÍAS FLORENZANO ${ }^{2}$, \\ VERÓNICA WOLFF ${ }^{1,3}$, JUAN CARLOS RODRÍGUEZ ${ }^{2}$, \\ HUGO VALENZUELA ${ }^{2}$, CRISTINA FERNÁNDEZ ${ }^{2}$, \\ ÁLVARO UNDURRAGA ${ }^{2}$

\section{Update on interstitial lung diseases}

Interstitial lung diseases are a broad, diverse, challenging group of diseases, most of them chronic whose prognosis is not good. In the last two decades there have been considerable advances in the knowledge of the epidemiology, pathological and genetic bases and treatment of several of these diseases. This article summarizes and presents updated information about their classification, new knowledge on genetics and treatments in idiopathic pulmonary fibrosis, advances in the diagnosis and management of hypersensitivity pneumonitis and a review of the broad spectrum of interstitial diseases associated with connective tissue diseases. Several clinical trials are currently underway whose results will be available in the coming years and will provide more information and tools to improve the treatment of these patients.

(Rev Med Chile 2019; 147: 1458-1467)

Key words: Alveolitis, Extrinsic Allergic; Idiopathic Interstitial Pneumonias; Idiopathic Pulmonary Fibrosis; Lung Disease, Interstitial.
L as enfermedades pulmonares intersticiales son un grupo heterogéneo de patologías caracterizadas por el compromiso difuso del tejido pulmonar, en su mayoría con importante morbimortalidad asociada ${ }^{1}$.

La importancia creciente en clínica e investigación de estas enfermedades queda reflejada en la numerosa cantidad de guías y consensos publicados por la Sociedad Americana de Tórax (ATS) desde el 2002 a la fecha: dos guías sobre la clasificación de las enfermedades intersticiales ${ }^{2,3}$, tres guías sobre diagnóstico y/o manejo de fibrosis pulmonar idiopática ${ }^{4-6}$, dos documentos sobre diagnóstico y manejo de linfangioleiomiomatosis (LAM $)^{7,8} y$ dos declaraciones sobre enfermedades autoinmunes ${ }^{9,10}$.

En este contexto, el objetivo de este artículo es dar una visión panorámica del estado actual de la numerosa cantidad de información nueva disponible y de las modificaciones recientes en las clasificaciones, en base a la experiencia de un grupo de médicos chilenos dedicado al tratamiento de este tipo enfermedades.

Para la mayor parte de las clasificaciones y nomenclaturas se utilizará la abreviatura usual en inglés, ya que es la más difundida en la literatura médica.

\section{Clasificación de las neumonías intersticiales idiopáticas}

Tradicionalmente las enfermedades intersticiales se han clasificado según grupos etiológicos, como son: relacionadas a enfermedades autoinmunes, causadas por agentes externos, un gran grupo de misceláneas y las neumonías intersticiales idiopáticas, para aquellas que se desconoce su causa. Probablemente uno de los puntos de mayor confusión, es que los patrones 
histológicos son compartidos entre enfermedades distintas, como, por ejemplo: el patrón histológico de Neumonia Intersticial Usual (UIP) subyace tanto a la fibrosis pulmonar idiopática como a un porcentaje de pacientes con artritis reumatoidea y esclerodermia.

La clasificación actualmente en uso de las neumonías intersticiales idiopáticas (NII) data de 2013 y se resumen en la Tabla $1^{2}$. Ésta propone seis NII mayores y dos enfermedades raras, agregando además el término de enfermedad clasificable. Los cambios respecto a la guía de 2002 fueron: incluir en forma definitiva la Neumonía intersticial no específica (NSIP) idiopática, desplazar la Neumonía intersticial linfoide idiopática (LIP) a las condiciones raras, ya que la mayor parte de estas tiene una causa identificable y se agregó la Fibroelastosis pleuro-parenquimatosa, entidad que ha sido crecientemente descrita a partir del año $2005 .{ }^{11-13}$. Otra entidad mencionada es la neumonía en organización aguda fibrinoide ${ }^{14,15}$ (AFOP) que no se incluye en la clasificación de idiopáticas por el momento.

\section{Tabla 1. Clasificación de las NII 2013, ATS/ERS²}

\footnotetext{
Neumonías intersticiales idiopáticas mayores

Fibrosis pulmonar idiopática

Neumonía intersticial no específica (NSIP) idiopática

Bronquiolitis respiratoria con enfermedad pulmonar intersticial (BR-ILD)

Neumonía intersticial descamativa (DIP)

Neumonía en organización criptogénica (COP)

Neumonía intersticial aguda (AIP)

Neumonías intersticiales idiopáticas de rara ocurrencia

Neumonía intersticial linfoide (LIP) idiopática

Fibroelastosis pleuro-parenquimatosa idiopática

Neumonías intersticiales idiopáticas inclasificables*

*Esta categoría incluye:

1) información clínica, radiológica o patológica inadecuada;

2) Discordancia mayor entre información clínica, radiológica y patológica, lo que puede ocurrir en las siguientes situaciones:

a) terapia previa que altera el patrón radiológico o histológico (ejemplo: DIP luego de terapia con esteroides);

b) entidad nueva o variantes raras de enfermedades reconocidas que no han sido adecuadamente caracterizadas;

c) patrones diversos en la TAC o en la histología, lo que puede ocurrir en pacientes con NII.
}

\section{Enfoque diagnóstico de las enfermedades intersticiales}

Probablemente el mayor reto de estas enfermedades es hacer el diagnóstico correcto y preciso, frente a cada paciente. El enfoque inicial debe enfocarse en buscar mediante la historia clínica y el examen físico claves diagnósticas como son:

- Interrogar por el uso de drogas potencialmente neumotóxicas.

- Buscar exposición a antígenos productores de hipersensibilidad.

- La existencia de síntomas generales, que hacen pensar en enfermedades sistémicas, como sarcoidosis.

- Elementos clínicos propios de las enfermedades autoinmunes ${ }^{16}$.

La tomografía computada de alta resolución (TAC) es el pilar fundamental en el diagnóstico, describiéndose cada año nuevos signos y claves radiológicas para aproximarse a un diagnóstico preciso.

Las guías internacionales han propuesto clasificaciones de los hallazgos de la TAC, enfocadas principalmente en el diagnóstico o exclusión de Fibrosis pulmonar idiopática (FPI). La última propuesta de la ATS se resume en la Tabla $2^{5}$. Nótese que el patrón de la TAC esta denominado en relación con el patrón histológico de la FPI, que es llamado Neumonía Intersticial Usual (UIP en inglés).

Si bien estas clasificaciones y enfoques han representado considerables avances en el enfoque clínico de estos pacientes, es en los pacientes con diagnósticos alternativos a UIP donde se presentan los grandes desafíos diagnósticos y adquiere un rol mayor la biopsia pulmonar.

Cuando la información clínica y el patrón de TAC no son diagnósticos se debe considerar la biopsia pulmonar por vía quirúrgica que es el estándar del punto de vista histológico, especialmente si la sospecha es FPI o NSIP ${ }^{25,26}$. La biopsia transbronquial puede permitir el diagnóstico de otras entidades como la sarcoidosis y la neumonía en organización, pero difícilmente permite diagnosticar FPI. El papel de la criobiopsia es controvertido, con estudios no aleatorizados que muestran rendimientos diagnósticos variables y complicaciones asociadas ${ }^{27-29}$. 
Tabla 2. Características de los patrones en la tomografía computada de alta resolución²

\begin{tabular}{|c|c|c|c|}
\hline UIP & Probable UIP & $\begin{array}{l}\text { Indeterminado } \\
\text { para UIP }\end{array}$ & Diagnósticos alternativos \\
\hline $\begin{array}{l}\text { Predominio } \\
\text { subpleural } \\
\text { y basal; la } \\
\text { distribución } \\
\text { es a menudo } \\
\text { heterogénea }\end{array}$ & $\begin{array}{l}\text { Predominio } \\
\text { subpleural } \\
\text { y basal; la } \\
\text { distribución } \\
\text { es a menudo } \\
\text { heterogénea }\end{array}$ & $\begin{array}{l}\text { Predominio } \\
\text { subpleural y basal }\end{array}$ & \multirow{3}{*}{$\begin{array}{l}\text { Existen hallazgos sugerentes de otros diagnósticos, incluyendo: } \\
\text { Características al TAC } \\
\text { Quistes } \\
\text { Atenuación en mosaico marcada } \\
\text { Predominio de vidrio esmerilado } \\
\text { Micro-nódulos profusos } \\
\text { Nódulos centro lobulillares } \\
\text { Nódulos } \\
\text { Consolidación } \\
\text { Distribución predominante } \\
\text { Peri-broncovascular } \\
\text { Peri-linfática } \\
\text { Lóbulos superiores o medios } \\
\text { Otros: } \\
\text { Placas pleurales (considerar asbestosis) } \\
\text { Esófago dilatado (considerar enfermedad del mesénquima) } \\
\text { Erosiones en clavículas distales (considerar artritis reumatoide) } \\
\text { Compromiso ganglionar extenso } \\
\text { Derrame o engrosamiento pleural (considerar enfermedades } \\
\text { del mesénquima o fármacos) }\end{array}$} \\
\hline \multirow[t]{2}{*}{$\begin{array}{l}\text { Existe panal } \\
\text { con o sin } \\
\text { bronquiectasias } \\
\text { por tracción o } \\
\text { bronquioloec- } \\
\text { tasias }\end{array}$} & $\begin{array}{l}\text { Patrón reticular } \\
\text { con bron- } \\
\text { quiectasias por } \\
\text { tracción o bron- } \\
\text { quioloectasias }\end{array}$ & $\begin{array}{l}\text { Sutil reticulación; } \\
\text { puede haber } \\
\text { mínimo vidrio } \\
\text { esmerilado o } \\
\text { distorsión (Ilamado } \\
\text { patrón UIP } \\
\text { temprano) }\end{array}$ & \\
\hline & $\begin{array}{l}\text { Puede haber } \\
\text { mínimo vidrio } \\
\text { esmerilado }\end{array}$ & $\begin{array}{l}\text { Las características } \\
\text { a la TAC o la } \\
\text { distribución de } \\
\text { la fibrosis no } \\
\text { sugieren ninguna } \\
\text { etiología específica }\end{array}$ & \\
\hline
\end{tabular}

La biopsia pulmonar quirúrgica no se recomienda en pacientes con deterioro pulmonar avanzado, usualmente DLCO bajo 35\% y CVF bajo $55 \%$ o en aquellos ventilados e inmunocomprometidos ${ }^{30}$. En pacientes de edad muy avanzada o con comorbilidades importantes debe valorarse cuidadosamente la obtención de biopsia por vía quirúrgica, con relación al riesgo y los beneficios potenciales.

Es importante enfatizar que la biopsia por sí sola no es diagnóstica en estas enfermedades e incluso con todos los elementos de juicio disponibles, un porcentaje de los pacientes quedará en la categoría inclasificable. El elemento más importante en el diagnóstico correcto de todas las enfermedades intersticiales es la discusión multidisciplinaria (neumólogo, patólogo, radiólogo y reumatólogo) que ha demostrado incrementar la precisión diagnóstica ${ }^{17}$. El comité multidisciplinario es el estándar de oro para el diagnóstico de las enfermedades pulmonares intersticiales.

\section{Fibrosis pulmonar idiopática}

La fibrosis pulmonar idiopática (FPI) es la patología más importante en este tipo de enfermedades por su prevalencia y pronóstico. Datos epidemiológicos recientes muestran que el número de enfermos va en aumento, aunque es posible que también exista mayor reconocimiento de la enfermedad y/o que se diagnostique en etapas más precoces ${ }^{18,19}$.

Durante muchos años se pensó que la inflamación era el mecanismo patogénico fundamental de la FPI hasta que a principios de siglo se planteó que la enfermedad se producía por una anómala cicatrización, en respuesta a repetidos microtraumas, en el epitelio alveolar, activando la formación de focos de fibroblastos y de miofibroblastos, los que llevan a la fibrosis ${ }^{20}$. Esta nueva concepción de la patogenia de la enfermedad dio paso a un nuevo enfoque en la búsqueda de terapias.

Aunque la fisiopatología de la enfermedad aun no es completamente comprendida, han habido avances en el conocimiento de la genética, identificado diversas alteraciones patogénicas relacionadas a acortamiento de telómeros, proteínas del surfactante y mecanismos de defensa celular, entre otros $^{21,22}$. Un polimorfismo identificado en el gen MUC5B, está presente en $40 \%$ de los pacientes con $\mathrm{FPI}^{23}$; siendo la alteración genética más asociada con esta enfermedad. Paradójicamente, los pacientes con FPI y esta mutación parecen tener una evolución más lenta en su enfermedad ${ }^{24}$. 
Existen actualmente dos fármacos disponibles para el tratamiento de FPI, los denominados anti-fibróticos: nintedanib y pirfenidona. Ambos medicamentos disminuyen la progresión de la enfermedad en alrededor de 50\% y algunos estudios recientes destacan que mejora la supervivencia de estos pacientes $^{31,32}$. Además de ello y muy relevante son las estrategias de tratamiento no farmacológico: cese de tabaco, uso de oxígeno según guías, vacunación y rehabilitación respiratoria ${ }^{4}$. El rol del reflujo gastroesofágico y su tratamiento no está del todo claro, existiendo datos que muestran beneficios y otros que dan cuenta de efectos adversos del uso de anti-ácidos ${ }^{33-35}$. Estudios en curso sobre terapia farmacológica y cirugía anti-reflujo debieran resolver esas dudas. A la luz de la información actual parece recomendable tratar con fármacos solamente a aquellos sujetos con reflujo sintomático y analizarlo caso a caso.

Para la enfermedad en etapas avanzadas la única opción es el trasplante pulmonar, siendo FPI la primera causa de trasplante en Chile ${ }^{36}$.

Casi todas los registros muestran una mortalidad alta a cinco años y un pronóstico ominoso, aunque en la práctica clínica es frecuente ver pacientes con supervivencias mucho mayor que lo descrito ${ }^{16}$. El único estudio nacional publicado da cuenta de una supervivencia un poco mejor a lo reportado a nivel internacional ${ }^{37}$.

Un concepto emergente es el de las anormalidades intersticiales pulmonares (ILA abreviado en inglés) apuntando a alteraciones diversas, de poca extensión, encontradas en la TAC de tórax de pacientes sin diagnóstico clínico, usualmente en el contexto de investigación o estudios por otros motivos que NII. El concepto de ILA intenta aproximarse a la FPI en etapas muy tempranas, pero está en evolución y se han usado distintas definiciones en los estudios publicados. Las investigaciones hasta ahora muestran que algunos de estos pacientes evolucionaran a FPI pero falta conocimiento para poder definir donde y cuando intervenir en este tipo de enfermos ${ }^{38,39}$.

\section{Neumonitis por hipersensibilidad (NH)}

La neumonitis por hipersensibilidad puede tener un perfil agudo o subagudo / crónico, siendo el primer caso de presentación clínica y radiológica muy distinta de los otros. En el contexto de este artículo nos referiremos solamente a las formas subagudas y crónicas que suelen solaparse.

De diagnóstico tanto o más difícil que la FPI, la $\mathrm{NH}$ es una enfermedad que no fue considerada relevante hasta principios de siglo, donde se comenzó a identificar en forma frecuente ${ }^{40}$. Datos recientes dan cuenta de su importancia: en el registro de enfermedades intersticiales en India, sobre 1.084 pacientes, la mitad de los casos corresponden a $\mathrm{NH}^{41}$ y un análisis de registros de defunción en USA da cuenta del aparente incremento en la mortalidad por $\mathrm{NH}$ en los últimos 20 años $^{42}$.

La NH es producida por una respuesta de hipersensibilidad mediada por linfocitos a sustancias habitualmente inhaladas y típicamente orgánicas; la cantidad de sustancias potencialmente productoras de $\mathrm{NH}$ es extensa y continúa incrementándose. Los antígenos más frecuentemente asociados varían según la población estudiada en base a características diversas como variables climáticas y geográficas ${ }^{43}$. En nuestro medio la causa más frecuente parece ser la exposición a antígenos aviares $^{44}$.

El diagnóstico de $\mathrm{NH}$ enfrenta diversos problemas: la identificación del antígeno causal con frecuencia no se logra, lo que además conlleva mal pronóstico ${ }^{45}$; la tomografía computada puede ser muy sugerente, pero los hallazgos son altamente variables $^{46}$. El lavado broncoalveolar cuando tiene predominio de linfocitos es orientador, aunque no está claramente definido el umbral de linfocitosis de mejor rendimiento diagnóstico, sugiriéndose usualmente 30 a $40 \%{ }^{43}$. Con frecuencia se utiliza la detección de precipitinas (anticuerpos) en sangre para antígenos causales posibles, lo cual tiene problemas de inadecuada sensibilidad, especificidad y falta de estandarización ${ }^{47}$. Se ha propuesto hacer una prueba de provocación con el antígeno causal, pero son pocos los centros que la realizan en el mundo y también adolece de problemas de estandarización ${ }^{43}$. Por último, está la biopsia pulmonar, que tampoco entrega certeza absoluta, ya que puede presentar mezcla de patrones histológicos ${ }^{48}$.

Para el diagnóstico de NH no existe un estándar universal, fundamentándose éste en la constelación de elementos de la historia, la tomografía y alguna de los otras herramientas nombradas en el párrafo previo. En ausencia de criterios diagnósticos universales, existen algunas recomendaciones de algoritmos ${ }^{46} \mathrm{y}$ un consenso de expertos publicado recientemente ${ }^{49}$. 
Los pacientes con $\mathrm{NH}$ suelen tener un deterioro progresivo, aunque se los retire de la exposición. No existen ensayos clínicos sobre tratamiento para esta enfermedad, pero varios estudios en desarrollo con drogas anti-fibróticas están incluyendo pacientes con NH. Se suele utilizar con frecuencia tratamientos con prednisona y azatioprina o micofenolato, existiendo estudios no aleatorizados que muestran resultados diversos e incluso que podría ser mejor no tratarlos ${ }^{50,51}$. Al igual que en FPI, el trasplante pulmonar es una opción en enfermedad avanzada.

\section{Enfermedad intersticial asociada a enfermedad del tejido conectivo (EI-ETC)}

El compromiso pulmonar en las ETC es un problema creciente. Mientras la mortalidad global por ETC parece venir disminuyendo ${ }^{52,53}$, datos actualizados de registros, cohortes y seguros muestran que el compromiso pulmonar en estos pacientes puede estar aumentado e incrementa la morbimortalidad de los enfermos ${ }^{54-56}$.

Si bien todas las enfermedades del mesénquima pueden comprometer el pulmón, las tres más relevantes por frecuencia y gravedad son las miopatías inflamatorias (incluyendo síndromes anti-sintetasa), la artritis reumatoide (AR) y esclerosis sistémica (ES). Un resumen de cada una de ellas se presenta en la Tabla 3.

Los mecanismos patogénicos subyacentes a estas enfermedades varían y no están del todo establecido. Interesantemente, estudios genéticos muestran que pacientes con AR presentan mutaciones similares a los pacientes con FPI, planteando una posible vía patogénica en común ${ }^{57,58}$.

El diagnóstico de EI-ETC puede resultar fácil en pacientes con ETC conocida que desarrollan un patrón radiológico típico como por ejemplo el compromiso pulmonar de patrón NSIP o UIP en pacientes con AR o ES. Sin embargo, algunos pacientes debutan con la enfermedad pulmonar o presentan elementos clínicos incompletos de la conectivopatía, en cuyo caso es fácil equivocarse.

El patrón tomográfico de la enfermedad pulmonar nuevamente es una pieza clave en el diagnóstico y es importante que haga sentido con la ETC que se está planteando.

Las diversas serologías y anticuerpos disponibles en el laboratorio ayudan, pero se debe tener presente que éstos no hacen diagnóstico sin los elementos clínicos y no son del todo específicos. Existen reportes sobre el frecuente hallazgos de anticuerpos en población general y en pacientes con FPI cuyo significado no es del todo claro ${ }^{59}$.

La visión bajo microscopio de los capilares del lecho ungueal ha emergido como un examen que puede ayudar en el diagnóstico diferencial de estos pacientes ${ }^{60}$. La biopsia pulmonar usualmente no se utiliza y rara vez aporta más información o cambia el manejo en el contexto de EI-ETC.

El pronóstico de las EI es muy variable entre las distintas ETC. El tratamiento considera el uso de esteroides e inmunosupresores. Entre los factores más importantes a considerar para iniciar terapia inmunosupresora están la severidad clínica (ejemplo: presentación aguda o subaguda y/o formas inflamatorias como NSIP o Neumonía en organización), la extensión radiológica del compromiso pulmonar (especialmente en ES), el deterioro funcional progresivo y las manifestaciones extrapulmonares que requieran tratamiento específico. En concordancia con lo anterior, los tratamientos son muy diversos y largamente basados en recomendaciones de expertos. Existen muchos fármacos inmunosupresores disponibles ${ }^{61,62}$, pero pocos estudios de calidad que permitan hacer recomendaciones basadas en buena evidencia.

Una excepción a lo anterior se da en el caso de ES, donde existen dos ensayos clínicos de terapia con ciclofosfamida y micofenolato que demostraron estabilizar la enfermedad ${ }^{63,64}$. Otros inmunosupresores disponibles son azatioprina, ciclosporina y recientemente tacrolimus, este último cada vez más utilizado en contexto de falla respiratoria grave. El rituximab suele utilizarse como segunda línea de tratamiento en casos graves, refractarios o con reacciones adversas a inmunosupresores, aunque también se ha planteado como estabilizador de enfermedad en casos menos graves ${ }^{65-67}$.

En pacientes con AR o ES y compromiso pulmonar de tipo UIP también se han usado inmunosupresores, pero a la luz del conocimiento disponible parece interesante tratarlos con anti-fibróticos. Recientemente se ha publicado el primer estudio de estos, demostrando efectividad de nintedanib en pacientes con esclerodermia, aunque la magnitud del efecto es pequeña ${ }^{68}$. Otros ensayos clínicos en desarrollo nos darán más claridad respecto al uso de estos fármacos. 
Tabla 3. Enfermedades pulmonares intersticiales relacionadas a enfermedades del tejido conectivo

\begin{tabular}{|c|c|c|c|}
\hline & Artritis reumatoide & $\begin{array}{l}\text { Esclerosis sistémica } \\
\text { progresiva (ESP) }\end{array}$ & $\begin{array}{l}\text { Miopatías inflamatorias } \\
\text { idiopáticas (MII) }\end{array}$ \\
\hline Prevalencia de EPI* & $\begin{array}{l}\text { Enfermedad clínica } 40 \% \\
\text { Alteraciones imágenes }>50 \%{ }^{78}\end{array}$ & $50 \%$ o más 79 & Hasta $78 \% 80$ \\
\hline $\begin{array}{l}\text { Factores/grupos de } \\
\text { riesgo descritos }\end{array}$ & $\begin{array}{l}\text { Tabaquismo } \\
\text { Género masculino }\end{array}$ & $\begin{array}{l}\text { Compromiso cutáneo difuso } \\
\text { Anticuerpos anti topoisomerasa } \\
\text { (SCL 70) } \\
\text { Raza afroamericana } \\
\text { Primeros } 5 \text { años luego } \\
\text { de diagnóstico de ESP }\end{array}$ & $\begin{array}{l}\text { Anticuerpos anti sintetasa } \\
\text { Anti MDA5 }\end{array}$ \\
\hline Serologías & $\begin{array}{l}\text { Factor reumatoide (FR) } \\
\text { Peptido citrulinado (CCP) }\end{array}$ & $\begin{array}{l}\text { ANA patrón nucleolar } \\
\text { SCL70 } \\
\text { PM-SCL } 75 \\
\text { PM-SCL100 (sobreposición con MI) }\end{array}$ & $\begin{array}{l}\text { JO-1; PL12; PL7; OJ; EJ } \\
\text { PM-SCL75; PM-SCL100; Ku } \\
\text { MDA5 } \\
\text { RO52\# }\end{array}$ \\
\hline $\begin{array}{l}\text { Elementos clínicos } \\
\text { claves }\end{array}$ & Poliartritis manos & $\begin{array}{l}\text { Esclerodactilia } \\
\text { Fenómeno de Raynaud } \\
\text { Úlceras de los pulpejos } \\
\text { Telangectasias periungueales } \\
\text { Telangectasias faciales y/o } \\
\text { palmares } \\
\text { Reflujo gastroesofágico }\end{array}$ & $\begin{array}{l}\text { Fiebre } \\
\text { Debilidad muscular/miositis } \\
\text { Rash heliotropo/edema facial } \\
\text { Telangectasias periungueales } \\
\text { Raynaud/úlceras cutáneas } \\
\text { Manos de mecánico } \\
\text { Poliartritis }\end{array}$ \\
\hline $\begin{array}{l}\text { Tipo de EPI más } \\
\text { asociadas }\end{array}$ & $\begin{array}{l}\text { UIP } \\
\text { NSIP }\end{array}$ & $\begin{array}{l}\text { NSIP } \\
\text { UIP }\end{array}$ & $\begin{array}{l}\text { OP / NSIP } \\
\text { NSIP (acentuado en bases } \\
\text { pulmonares) } \\
\text { AIP }\end{array}$ \\
\hline
\end{tabular}

* Las prevalencias de EPI son altamente variables dependiendo del método de tamizado que se utilice, la etapa o definición de ETC usada, existiendo además variaciones epidemiológicas. Se intenta dar una aproximación a la relevancia del problema y se indica la referencia utilizada. "Este anticuerpo se asocia con miopatía inflamatorias, pero no es anticuerpo específico de miositis. Su rol exacto aún está por definirse. ANA: Anticuerpos antinucleares. OP: Neumonia en organización. NSIP: Neumonia intersticial no específica. AIP: Neumonia intersticial aguda. UIP: Neumonia intersticial usual.

Al igual que en las otras patologías, el trasplante pulmonar es una opción en pacientes con enfermedad avanzada. Existe controversia respecto al trasplante de pacientes con ES y compromiso de la motilidad esofágica por posible mayor riesgo asociado de rechazo crónico del injerto pulmonar ${ }^{69,70}$. En estos pacientes el trasplante de progenitores hematopoyéticos puede ser una opción terapéutica ${ }^{71}$.

En el año 2015 la sociedad americana de tórax publicó un documento proponiendo un concepto nuevo, con fines de investigación, denominado Neumonía Intersticial con Características Autoinmunes (IPAF) intentando agrupar, ordenar y dar espacio a pacientes que presentan enfermedad intersticial y pueden presentar elementos sugerentes de autoinmunidad aunque no un diagnóstico reumatológico concreto ${ }^{9}$. Su principal aporte ha sido crear conciencia en relación a la existencia de formas incompletas de ETC que se inician o mantienen afectando en forma predominante el pulmón y manteniendo al equipo clínico alerta acerca de la posibilidad de considerar terapia inmunomoduladora en estos pacientes. El concepto de IPAF se encuentra en evolución y no constituye una enfermedad en sí, existiendo cierta controversia aún respecto a su real aporte y utilidad en el ámbito clínico ${ }^{72,73}$

\section{Otras consideraciones}

La lista de enfermedades intersticiales es enorme y no es posible revisarlas todas en este documento; sin embargo, las patologías mencionadas 
son las que han experimentado más novedades y son las de frecuencias más importante en nuestro medio.

Otras enfermedades relevantes como la sarcoidosis o la linfangioleiomiomatosis (LAM) son infrecuentes en nuestro medio o se encuentran subdiagnosticadas. Una revisión reciente sobre la primera ha sido publicada recientemente ${ }^{74}$ y dos guías de diagnóstico y tratamiento de LAM están disponibles ${ }^{7,8}$.

En los últimos años también se ha agregado otro concepto que es el de Enfermedad Intersticial Fibrosante Progresiva (PF-ILD), que intenta agrupar a las enfermedades intersticiales distintas de la FPI, pero que presentan las siguientes características: un curso progresivo, grados variables de inflamación y fibrosis a nivel histológico y pobre respuesta a terapias inmunomoduladoras. Estas enfermedades incluyen entre otras: la NSIP idiopática, la neumonitis por hipersensibilidad crónica y algunas EPI-ETC como la UIP asociada a AR o esclerodermia. La racionalidad de agrupar estas enfermedades subyace en sus características comunes, lo que lleva a plantear una respuesta terapéutica similar a drogas nuevas del espectro antifibrótico $^{75}$.

Las NII de curso agudo como la neumonía intersticial aguda (AIP) y la neumonía en organización criptogénica (COP) tampoco han sido discutidas en este documento. Estas entidades con frecuencia se dan en un contexto distinto, hospitalario, de falla respiratoria aguda o de sospecha de infección ${ }^{76,77}$. La cantidad de investigación para estas entidades es escasa y es muy probable que ambas estén subdiagnosticadas.

\section{Comentario final}

Las enfermedades intersticiales se encuentran en este momento en un punto álgido de investigación científica y de desarrollo terapéutico. Diversas drogas nuevas se encuentran en estudio para estas enfermedades y otros fármacos ya conocidos también están probándose en estas enfermedades. Los tratamientos que actualmente disponemos están cambiando el histórico mal pronóstico de estas enfermedades. Probablemente en los próximos años tendremos varias opciones de terapias solas o combinadas, que contribuirán aun más a mejorar la calidad de vida de estos enfermos.

\section{Referencias}

1. Antoniou KM, Margaritopoulos GA, Tomassetti S, Bonella F, Costabel U, Poletti V. Interstitial lung disease. Eur Respir Rev 2014; 23 (131): 40-54.

2. Travis WD, Costabel U, Hansell DM, King TE, Lynch DA, Nicholson AG, et al. An official American Thoracic Society/European Respiratory Society statement: Update of the international multidisciplinary classification of the idiopathic interstitial pneumonias. Am J Respir Crit Care Med 2013; 188 (6): 733-48.

3. American Thoracic Society, European Respiratory Society. American Thoracic Society/European Respiratory Society International Multidisciplinary Consensus Classification of the Idiopathic Interstitial Pneumonias. Am J Respir Crit Care Med 2002; 165 (2): 277-304.

4. Raghu G, Collard HR, Egan JJ, Martínez FJ, Behr J, Brown KK, et al. An Official ATS/ERS/JRS/ALAT Statement: Idiopathic Pulmonary Fibrosis: Evidence-based Guidelines for Diagnosis and Management. Am J Respir Crit Care Med 2011; 183 (6): 788-824.

5. Raghu G, Remy-Jardin M, Myers JL, Richeldi L, Ryerson CJ, Lederer DJ, et al. Diagnosis of Idiopathic Pulmonary Fibrosis. An Official ATS/ERS/JRS/ALAT Clinical Practice Guideline. Am J Respir Crit Care Med 2018; 198 (5): e44-68.

6. Raghu G, Rochwerg B, Zhang Y, Garcia CAC, Azuma A, Behr J, et al. An Official ATS/ERS/JRS/ALAT Clinical Practice Guideline: Treatment of Idiopathic Pulmonary Fibrosis. An Update of the 2011 Clinical Practice Guideline. Am J Respir Crit Care Med 2015; 192 (2): e3-19.

7. Mccormack FX, Gupta N, Finlay GR, Young LR, Taveira-Dasilva AM, Glasgow CG, et al. Official American Thoracic Society/Japanese Respiratory Society Clinical Practice Guidelines: Lymphangioleiomyomatosis Diagnosis and Management. Am J Respir Crit Care Med 2016; 194 (6): 748-61.

8. Gupta N, Finlay GA, Kotloff RM, Strange C, Wilson KC, Young LR, et al. Lymphangioleiomyomatosis Diagnosis and Management: High-Resolution Chest Computed Tomography, Transbronchial Lung Biopsy, and Pleural Disease Management An Official American Thoracic Society/Japanese Respiratory Society. Am J Respir Crit Care Med 2017; 196 (10): 1337-48.

9. Fischer A, Antoniou KM, Brown KK, Cadranel J, Corte TJ, du Bois RM, et al. An official European Respiratory Society/American Thoracic Society research statement: interstitial pneumonia with autoimmune features. Eur Respir J 2015; 46 (4): 976-87.

10. White ES, Borok Z, Brown KK, Eickelberg O, Guenther A, Jenkins RG, et al. An American Thoracic Society 
Official Research Statement: Future Directions in Lung Fibrosis Research. Am J Respir Crit Care Med 2016; 193 (7): 192-800.

11. Frankel SK, Cool CD, Lynch DA, Brown KK. Idiopathic Pleuroparenchymal Fibroelastosis. Chest 2004; 126 (6): 2007-13.

12. Watanabe K. Pleuroparenchymal Fibroelastosis: Its Clinical Characteristics. Curr Respir Med Rev 2013; 9 (4): 299-37.

13. Shioya M, Otsuka M, Yamada G, Umeda Y, Ikeda K, Nishikiori H, et al. Poorer Prognosis of Idiopathic Pleuroparenchymal Fibroelastosis Compared with Idiopathic Pulmonary Fibrosis in Advanced Stage. Can Respir J 2018; 2018: 6043053.

14. Beasley MB, Franks TJ, Galvin JR, Gochuico B, Travis WD. Acute fibrinous and organizing pneumonia: a histological pattern of lung injury and possible variant of diffuse alveolar damage. Arch Pathol Lab Med 2002; 126 (9): 1064-70.

15. Matos Gomes R, Padrão E, Soares Pires F, Neves I, Mota $\mathrm{P}$, Melo N, et al. Acute fibrinous and organizing pneumonia: A retrospective study. In: 15 Diffuse Parenchymal Lung Disease. European Respiratory Society; 2015. p. PA817.

16. Lederer DJ, Martínez FJ. Idiopathic Pulmonary Fibrosis. Longo DL, editor. N Engl J Med 2018; 378 (19): 181123.

17. Walsh SLF, Wells AU, Desai SR, Poletti V, Piciucchi S, Dubini A, et al. Multicentre evaluation of multidisciplinary team meeting agreement on diagnosis in diffuse parenchymal lung disease: a case-cohort study. Lancet Respir Med 2016; 4 (7): 557-65.

18. Olson AL, Gifford AH, Inase N, Pérez ERF, Suda T. The epidemiology of idiopathic pulmonary fibrosis and interstitial lung diseases at risk of a progressive-fibrosing phenotype. Eur Respir Rev 2018; 27 (150): 180077.

19. Hutchinson J, Fogarty A, Hubbard R, McKeever T. Global incidence and mortality of idiopathic pulmonary fibrosis: a systematic review. Eur Respir J 2015; 46 (3): 795-806.

20. Selman M, King TE, Pardo A, American Thoracic Society, European Respiratory Society, American College of Chest Physicians. Idiopathic pulmonary fibrosis: prevailing and evolving hypotheses about its pathogenesis and implications for therapy. Ann Intern Med 2001; 134 (2): 136-51.

21. Spagnolo P, Cottin V. Genetics of idiopathic pulmonary fibrosis: from mechanistic pathways to personalised medicine. J Med Genet 2017; 54 (2): 93-9.

22. Noth I, Zhang Y, Ma S-F, Flores C, Barber M, Huang $\mathrm{Y}$, et al. Genetic variants associated with idiopathic pulmonary fibrosis susceptibility and mortality: a genome-wide association study. Lancet Respir Med 2013; 1 (4): 309-17.

23. Hunninghake GM, Hatabu H, Okajima Y, Gao W, Dupuis J, Latourelle JC, et al. MUC5B Promoter Polymorphism and Interstitial Lung Abnormalities. N Engl J Med 2013; 368 (23): 2192-200.

24. Peljto AL, Zhang Y, Fingerlin TE, Shwu-Fan M, Garcia JGN, Richards TJ, et al. Association between the MUC5B promoter polymorphism and survival in patients with idiopathic pulmonary fibrosis. JAMA - J Am Med Assoc 2013; 309 (21): 2232-9.

25. Leslie KO. My approach to interstitial lung disease using clinical, radiological and histopathological patterns. J Clin Pathol 2009; 62 (5): 387-401.

26. Leslie KO, Gruden JF, Parish JM, Scholand MB. Transbronchial biopsy interpretation in the patient with diffuse parenchymal lung disease. Arch Pathol Lab Med 2007; 131 (3): 407-23.

27. Tomassetti S, Wells AU, Costabel U, Cavazza A, Colby TV, Rossi G, et al. Bronchoscopic Lung Cryobiopsy Increases Diagnostic Confidence in the Multidisciplinary Diagnosis of Idiopathic Pulmonary Fibrosis. Am J Respir Crit Care Med 2016; 193 (7): 745-52.

28. Romagnoli M, Colby T V, Berthet J-P, Gamez AS, Mallet J-P, Serre I, et al. Poor Concordance between Sequential Transbronchial Lung Cryobiopsy and Surgical Lung Biopsy in the Diagnosis of Diffuse Interstitial Lung Diseases. Am J Respir Crit Care Med 2019; 199 (10): 1249-56.

29. Ravaglia C, Wells AU, Tomassetti S, Gurioli C, Gurioli C, Dubini A, et al. Diagnostic yield and risk/benefit analysis of trans-bronchial lung cryobiopsy in diffuse parenchymal lung diseases: a large cohort of 699 patients. BMC Pulm Med 2019; 19 (1): 16.

30. Han Q, Luo Q, Xie J-X, Wu L-L, Liao L-Y, Zhang X-X, et al. Diagnostic yield and postoperative mortality associated with surgical lung biopsy for evaluation of interstitial lung diseases: A systematic review and meta-analysis. J Thorac Cardiovasc Surg 2015; 149 (5): 1394-401.e1.

31. Nathan SD, Albera C, Bradford WZ, Costabel U, Glaspole I, Glassberg MK, et al. Effect of pirfenidone on mortality: pooled analyses and meta-analyses of clinical trials in idiopathic pulmonary fibrosis. Lancet Respir Med 2017; 5 (1): 33-41.

32. Richeldi L, Cottin V, du Bois RM, Selman M, Kimura $T$, Bailes $Z$, et al. Nintedanib in patients with idiopathic pulmonary fibrosis: Combined evidence from the TOMORROW and INPULSIS ${ }^{\circledR}$ trials. Respir Med 2016; 113: 74-9. 
33. Costabel U, Behr J, Crestani B, Stansen W, SchlenkerHerceg R, Stowasser S, et al. Anti-acid therapy in idiopathic pulmonary fibrosis: insights from the INPULSIS ${ }^{\circledR}$ trials. Respir Res 2018; 19 (1): 167.

34. Johannson KA, Strâmbu I, Ravaglia C, Grutters JC, Valenzuela C, Mogulkoc N, et al. Antacid therapy in idiopathic pulmonary fibrosis: more questions than answers? Lancet Respir Med 2017; 5 (7): 591-8.

35. Lee JS, Collard HR, Anstrom KJ, Martínez FJ, Noth I, Roberts RS, et al. Anti-acid treatment and disease progression in idiopathic pulmonary fibrosis: an analysis of data from three randomised controlled trials. Lancet Respir Med 2013; 1 (5): 369-76.

36. Parada CMT, Melo TJ, Sepúlveda LC, Lazo PD, Linacre SV, Villalabeitía RE, et al. Lung transplantation in 87 patients with idiopathic pulmonary fibrosis. Rev Chil Enfermedades Respir 2015; 31 (4).

37. Salinas M, Florenzano M, Sabbagh E, Meneses M, Fernández C, Jalilie A, et al. Supervivencia de pacientes con fibrosis pulmonar idiopática diagnosticados por biopsia quirúrgica de pulmón: experiencia del Instituto Nacional del Tórax. Rev Med Chile 2014; 142 (1): 9-15.

38. Wells AU, Kokosi MA. Subclinical Interstitial Lung Abnormalities: Toward the Early Detection of Idiopathic Pulmonary Fibrosis? Am J Respir Crit Care Med 2016; 194 (12): 1445-6.

39. Araki T, Putman RK, Hatabu H, Gao W, Dupuis J, Latourelle JC, et al. Development and Progression of Interstitial Lung Abnormalities in the Framingham Heart Study. Am J Respir Crit Care Med 2016; 194 (12): 1514-22.

40. Churg A, Ryerson CJ. The Many Faces of Hypersensitivity Pneumonitis. Chest 2017; 152 (3): 458-60.

41. Singh S, Collins BF, Sharma BB, Joshi JM, Talwar D, Katiyar S, et al. Interstitial Lung Disease in India. Results of a Prospective Registry. Am J Respir Crit Care Med 2017; 195 (6): 801-13.

42. Fernández Pérez ER, Sprunger D, Ratanawatkul P, Maier LA, Huie TJ, Swigris JJ, et al. Increasing Hypersensitivity Pneumonitis-Related Mortality in the United States from 1988 to 2016. Am J Respir Crit Care Med; 199 (10): 1284-7.

43. Morell F, Villar A, Ojanguren I, Muñoz X, Cruz M-J. Hypersensitivity Pneumonitis: Challenges in Diagnosis and Management, Avoiding Surgical Lung Biopsy. Semin Respir Crit Care Med 2016; 37 (03): 395-405.

44. Salinas M, Fritz R, Meneses M, Díaz JC, Rodríguez JC, Florenzano M, et al. Histopathological findings of biopsies from patients with extrinsic allergic alveolitis (hipersensitivity pneumonia) in subacute or chronic phase. In: European Respiratory Journal. ERS Journals;
2013. p. P3811.

45. Fernández Pérez ER, Swigris JJ, Forssén AV, Tourin O, Solomon JJ, Huie TJ, et al. Identifying an Inciting Antigen Is Associated With Improved Survival in Patients With Chronic Hypersensitivity Pneumonitis. Chest 2013; 144 (5): 1644-51.

46. Selman M, Pardo A, King TE. Hypersensitivity Pneumonitis. Am J Respir Crit Care Med 2012; 186 (4): 314-24.

47. Kouranos V, Jacob J, Nicholson A, Renzoni E. Fibrotic Hypersensitivity Pneumonitis: Key Issues in Diagnosis and Management. J Clin Med 2017; 6 (6).

48. Trahan S, Hanak V, Ryu JH, Myers JL. Role of Surgical Lung Biopsy in Separating Chronic Hypersensitivity Pneumonia From Usual Interstitial Pneumonia/Idiopathic Pulmonary Fibrosis ${ }^{\star}$ : Analysis of 31 Biopsies From 15 Patients. Chest 2008; 134 (1): 126-32.

49. Morisset J, Johannson KA, Jones KD, Wolters PJ, Collard HR, Walsh SLF, et al. Identification of Diagnostic Criteria for Chronic Hypersensitivity Pneumonitis. An International Modified Delphi Survey. Am J Respir Crit Care Med 2018; 197 (8): 1036-44.

50. Adegunsoye A, Oldham JM, Fernández Pérez ER, Hamblin M, Patel N, Tener M, et al. Outcomes of immunosuppressive therapy in chronic hypersensitivity pneumonitis. ERJ open Res 2017; 3 (3).

51. Morisset J, Johannson KA, Vittinghoff E, Aravena C, Elicker BM, Jones $\mathrm{KD}$, et al. Use of Mycophenolate Mofetil or Azathioprine for the Management of Chronic Hypersensitivity Pneumonitis. Chest 2017; 151 (3): 61925.

52. Santo AH, Souza JMP, Pinheiro CE, Souza DC, Sato EI. Trends in dermatomyositis- and polymyositis-related mortality in the state of São Paulo, Brazil, 1985-2007: multiple cause-of-death analysis. BMC Public Health 2010; 10 (1): 597.

53. Dadoun S, Zeboulon-Ktorza N, Combescure C, Elhai M, Rozenberg S, Gossec L, et al. Mortality in rheumatoid arthritis over the last fifty years: Systematic review and meta-analysis. Jt Bone Spine 2013; 80 (1): 29-33.

54. Raimundo K, Solomon JJ, Olson AL, Kong AM, Cole $\mathrm{AL}$, Fischer A, et al. Rheumatoid Arthritis-Interstitial Lung Disease in the United States: Prevalence, Incidence, and Healthcare Costs and Mortality. J Rheumatol 2019; 46 (4): 360-9.

55. Sánchez-Cano D, Ortego-Centeno N, Callejas JL, Fonollosa Plá V, Ríos-Fernández R, Tolosa-Vilella C, et al. Interstitial lung disease in systemic sclerosis: data from the spanish scleroderma study group. Rheumatol Int 2018; 38 (3): 363-74.

56. Narváez J, Borrell H, Sánchez-Alonso F, Rúa-Figueroa I, López-Longo FJ, Galindo-Izquierdo M, et al. Primary 
respiratory disease in patients with systemic lupus erythematosus: data from the Spanish rheumatology society lupus registry (RELESSER) cohort. Arthritis Res Ther 2018; 20 (1): 280.

57. Juge P-A, Lee JS, Ebstein E, Furukawa H, Dobrinskikh E, Gazal S, et al. MUC5B Promoter Variant and Rheumatoid Arthritis with Interstitial Lung Disease. N Engl J Med 2018; 379 (23): 2209-19.

58. Juge P-A, Borie R, Kannengiesser C, Gazal S, Revy P, Wemeau-Stervinou L, et al. Shared genetic predisposition in rheumatoid arthritis-interstitial lung disease and familial pulmonary fibrosis. Eur Respir J 2017; 49 (5): 1602314.

59. Lee JS, Kim EJ, Lynch KL, Elicker B, Ryerson CJ, Katsumoto TR, et al. Prevalence and clinical significance of circulating autoantibodies in idiopathic pulmonary fibrosis. Respir Med 2013; 107 (2): 249-55.

60. Bouchard-Boivin M, Chartrand S, Koenig M, Joyal F, Assayag D, Morisset J. Role of Nailfold Capillaroscopy in the Evaluation of Patients With Interstitial Lung Disease. Chest 2017; 152 (4): A482.

61. Fischer A, Brown KK, Du Bois RM, Frankel SK, Cosgrove GP, Fernández-Pérez ER, et al. Mycophenolate Mofetil Improves Lung Function in Connective Tissue Disease-associated Interstitial Lung Disease. J Rheumatol 2013; 40 (5): 640-6.

62. Oldham JM, Lee C, Valenzi E, Witt LJ, Adegunsoye A, Hsu S, et al. Azathioprine response in patients with fibrotic connective tissue disease-associated interstitial lung disease. Respir Med 2016; 121: 117-22.

63. Tashkin DP, Elashoff R, Clements PJ, Goldin J, Roth $\mathrm{MD}$, Furst DE, et al. Cyclophosphamide versus Placebo in Scleroderma Lung Disease. N Engl J Med 2006; 354 (25): 2655-66.

64. Tashkin DP, Roth MD, Clements PJ, Furst DE, Khanna D, Kleerup EC, et al. Mycophenolate mofetil versus oral cyclophosphamide in scleroderma-related interstitial lung disease (SLS II): a randomised controlled, double-blind, parallel group trial. Lancet Respir Med 2016; 4 (9): 708-19.

65. Md Yusof MY, Kabia A, Darby M, Lettieri G, Beirne P, Vital EM, et al. Effect of rituximab on the progression of rheumatoid arthritis-related interstitial lung disease: 10 years' experience at a single centre. Rheumatology 2017; 56 (8): 1348-57.
66. Chartrand S, Swigris JJ, Peykova L, Fischer A. Rituximab for the treatment of connective tissue disease-associated interstitial lung disease. Sarcoidosis, Vasc Diffus lung Dis 2016; 32 (4): 296-304.

67. Lepri G, Avouac J, Airò P, Anguita Santos F, Bellando-Randone S, Blagojevic J, et al. Effects of rituximab in connective tissue disorders related interstitial lung disease. Clin Exp Rheumatol 2016; 34 Suppl 100 (5):181-5.

68. Distler O, Highland KB, Gahlemann M, Azuma A, Fischer A, Mayes MD, et al. Nintedanib for Systemic Sclerosis-Associated Interstitial Lung Disease. N Engl J Med 2019; 380 (26): 2518-28.

69. Shah RJ, Boin F. Lung Transplantation in Patients With Systemic Sclerosis. Curr Rheumatol Rep 2017; 19 (5): 23.

70. Pradère P, Tudorache I, Magnusson J, Savale L, Brugiere O, Douvry B, et al. Lung transplantation for scleroderma lung disease: An international, multicenter, observational cohort study. J Hear Lung Transplant 2018; 37 (7): 903-11.

71. Sullivan KM, Goldmuntz EA, Keyes-Elstein L, McSweeney PA, Pinckney A, Welch B, et al. Myeloablative Autologous Stem-Cell Transplantation for Severe Scleroderma. N Engl J Med 2018; 378 (1): 35-47.

72. Lee JS, Fischer A. POINT: Does Interstitial Pneumonia With Autoimmune Features Represent a Distinct Class of Patients With Idiopathic Interstitial Pneumonia? Yes. Chest 2019; 155 (2): 258-60.

73. Oldham JM, Danoff SK. COUNTERPOINT: Does Interstitial Pneumonia With Autoimmune Features Represent a Distinct Class of Patients With Idiopathic Interstitial Pneumonia? No. Chest 2019; 155 (2): 260-3.

74. Spagnolo P, Rossi G, Trisolini R, Sverzellati N, Baughman RP, Wells AU. Pulmonary sarcoidosis. Lancet Respir Med 2018; 6 (5): 389-402.

75. Cottin V, Hirani NA, Hotchkin DL, Nambiar AM, Ogura T, Otaola M, et al. Presentation, diagnosis and clinical course of the spectrum of progressive-fibrosing interstitial lung diseases. Eur Respir Rev 2018; 27 (150): 180076.

76. Disayabutr S, Calfee CS, Collard HR, Wolters PJ. Interstitial lung diseases in the hospitalized patient. BMC Med 2015; 13: 245.

77. Cordier J-F. Cryptogenic organising pneumonia. Eur Respir J 2006; 28 (2): 422-46. 